\title{
Impact of surgical technique on operative morbidity and its socioeconomic benefit in thyroid surgery
}

\author{
Anita Kurmann - Forian Martens • Roman Inglin • \\ Stefan W. Schmid - Daniel Candinas • Christian A. Seiler
}

Received: 9 November 2011 / Accepted: 4 July 2012 /Published online: 18 July 2012

(C) Springer-Verlag 2012

\begin{abstract}
Purpose The aim of this retrospective cohort study was to evaluate the rate of complications in relation to the extent of surgery and some of its consequences.

Methods Between 1972 and 2010, a total of 5,277 consecutive thyroid gland surgeries with 7,383 nerves at risk were performed at our teaching institution. Data of all patients undergoing thyroidectomy were collected prospectively. A total of 2,867 subtotal resections (first study period from 1972 to 1990 ) were compared with 2,410 extended thyroid resections involving at least a hemithyroidectomy (second period from 1991 to 2010).

Results The incidence of permanent recurrent laryngeal nerve palsy in primary operations was significantly higher in the first period compared to the second period (3.6 vs. $0.9 \% ; p<0.001)$. Permanent hypoparathyroidism decreased from $3.2 \%$ in the first period to $0.8 \%$ in the second period $(p<0.001)$. The incidence of recurrent goiter surgery decreased from $11.1 \%$ in the first period to $8.1 \%$ in the second period $(p<0.001)$. No significant difference was found in permanent recurrent laryngeal nerve palsy in recurrent disease between the two periods. The socioeconomic benefits of an extended thyroid resection in our patient population are 360 preventable operations, 90 preventable permanent recurrent laryngeal nerve palsies, and 58 preventable cancers. Furthermore, 37 preventable radioiodine ablations and 15 preventable deaths were associated with more radical thyroid resection.
\end{abstract}

This study was presented at the 98th Annual Congress of the Swiss Society of Surgery, May 2011, Geneva, Switzerland.

A. Kurmann · F. Martens · R. Inglin · S. W. Schmid •

D. Candinas $\cdot$ C. A. Seiler $(\bowtie)$

Department of Visceral Surgery and Medicine,

Inselspital University Hospital Bern, University of Bern,

CH-3010, Bern, Switzerland

e-mail: christian.a.seiler@insel.ch
Conclusion Improvements in surgical technique and change in surgical strategy significantly decreased the prevalence of recurrent laryngeal nerve palsy, hypoparathyroidism, and recurrent disease as well as reduced public health costs associated with recurrent goiter.

Keywords Thyroidectomy $\cdot$ Recurrence rate $\cdot$ Recurrent laryngeal nerve palsy $\cdot$ Hypoparathyroidism $\cdot$ Socioeconomic benefits

\section{Introduction}

Subtotal thyroidectomy or nodal enucleation has become the "gold standard" treatment for multinodular benign goiter since Theodor Kocher published his experiences in thyroid surgery over 100 years ago $[1,2]$. In addition to establishing these surgical principles, Theodor Kocher researched the etiology of multinodular goiter disease and introduced the addition of iodine to table salt in Switzerland in 1922. In spite of sufficient dietary iodine intake in the present day, multinodular goiter remains an endemic disease in Switzerland [3]. Further research and understanding of the molecular growth mechanism in goitrogenesis [4-6] and an inacceptable high recurrence rate associated with subtotal thyroidectomy led endocrine surgeons to adopt a more extended surgical resection for benign multinodular goiter disease [7-11]. Previous studies showed that total thyroid resection is a safe procedure. However, these studies included mostly small numbers of patients or a short observation period of less than 20 years [12-14].

Public health costs are primarily reflected in the costs of operative morbidity and redo surgery. However, cost analysis in thyroid surgery is mainly focused on length of hospital stay and surgical devices, among other issues $[15,16]$. There is no study investigating the socioeconomic benefits 
of a more radical thyroid resection. The aim of this retrospective cohort study was to evaluate morbidity rate and socioeconomic benefits in relation to the extent of surgery with a large prospective single institutional cohort database spanning over 39 years.

\section{Material and methods}

Patients

Between 1972 and 2010, a total of 5,277 consecutive thyroid gland surgeries with 7,383 nerves at risk were performed at our teaching institution with a special interest in endocrine surgery. Data of all patients undergoing thyroidectomy were collected prospectively in an electronic database and analyzed retrospectively. The primary aim of this database was to provide data for quality control of thyroid surgery at our institution. Questionnaires were completed up to 1 year after the operation and contained information about patients' demographic parameters, operative procedures, intraoperative complications, and local and general postoperative complications. Overall loss to follow-up was $1 \%$.

In the present analysis, two study periods were compared. In the first study period from 1972 to 1990 , patients underwent subtotal resections. In the second study period from 1991 to 2010, extended resections involving at least hemithyroidectomy or total thyroidectomy were performed.

\section{Methods}

According to Kocher's principles [1, 2], during the first study period from 1972 to 1990 , a total of 2,867 patients underwent subtotal thyroidectomy (leaving at least 4-8 ml of thyroid tissue in place) or nodal enucleations of thyroid nodules in order to avoid thyroid hormone substitution.

In the second study period from 1991 to 2010, a total of 2,410 patients were included. A hemithyroidectomy was performed in patients with strictly unilateral thyroid disease. A total thyroidectomy was performed in cases of cancer or when nodules were present in both thyroid lobes. In cases of malignancy, lymphadenectomy was performed accordingly.

In our teaching hospital, the operations were performed by residents following a strict protocol; however, a staff surgeon was present in all operations in both study periods. Standardized visualization of the recurrent laryngeal nerves and the parathyroid glands was performed routinely in all operations in the second study period from 1991 to 2010. Furthermore, neuromonitoring was used routinely in the second study period since 2003 [17]. Removed tissue was submitted for intraoperative frozen section analysis, and histological analyses were performed in all patients.
Persistent recurrent laryngeal nerve palsy was defined as nerve palsy lasting more than 12 months after the operation. Vocal cord function was documented pre- and postoperatively by laryngoscopy in all patients. If postoperatively recurrent laryngeal nerve palsy was observed, patients underwent phoniatric consultations and logotherapy and were followed for 1 year.

Persistent hypoparathyroidism was defined as hypocalcemia lasting more than 12 months after surgery. Transplantation of parathyroid glands into the sternocleidomastoid muscle was performed if a parathyroid gland was devascularized during the operation. General practitioners were contacted by phone 1 year after the operation if a prescription of vitamin $\mathrm{D}$ and calcium was given at discharge.

\section{Model of cost analysis}

Operative and in-hospital costs for thyroidectomy were assessed on the basis of the Swiss All Patient DiagnosisRelated Groups (APDRG) 2010 (total costs, 8,190.20 EUR). The median salary of an inhabitant per month in Switzerland in 2008 was 4,512.93 EUR referred to the federal office for statistics in Switzerland. With a median lack of work after thyroidectomy of 10 days, a loss of salary of 1,504.31 EUR per patient was calculated. Median cost of 1,278.78 EUR per patient for recurrent laryngeal nerve palsy therapy including at least three phoniatric consultations and 12 logotherapy sessions was calculated. Costs of radioiodine ablation therapy were also based on the Swiss APDRG and resulted in 5,010.38 EUR per case. All results are presented in Euros (€) using values for 2011 ( $1 \mathrm{CHF}=0.775 \mathrm{EUR})$.

\section{Outcome parameters}

The primary outcome parameter of this study was permanent recurrent laryngeal nerve palsy in relation to the extent of surgery. Secondary outcome measures were permanent hypoparathyroidism, recurrence rate, and total cost of redo surgery.

Statistical analysis

Student's $t$ test was performed to determine the significance between continuous variables. Categorical variables were compared using Fisher's exact test. One-way ANOVA was used to analyze linear regression models. $P$ values were two-sided, and $p<0.05$ was considered statistically significant (NCSS 2007 for Windows; NCSS, Kaysville, UT).

\section{Results}

A total of 5,277 patients with 7,383 nerves at risk underwent thyroid gland surgery between 1972 and 2010 at our 
institution and were included in this study. Subtotal resections were performed in 2,867 cases (first study period), and extended thyroid resections involving at least hemithyroidectomy were performed in 2,410 cases (second study period). In the second study period, 925 patients $(38.4 \%)$ underwent hemithyroidectomy, and 1,485 (61.6\%) patients underwent total thyroidectomy. Demographic parameters including age and sex were not significantly different between groups. Table 1 reports indications for primary and redo operation. A total of 196 patients who received redo surgery in the second study period had had primary operation during the first period or subtotal resection elsewhere.

Table 2 shows long-term outcomes after thyroid surgery between the two study groups. The incidence of permanent recurrent laryngeal nerve palsy with primary operations was significantly higher in the first period compared to the second period ( 3.6 vs. $0.9 \% ; p<0.001$ ). Bilateral nerve palsy was significantly higher in the first period compared to the second period ( 2.3 vs. $0.13 \% ; p<0.001)$. Permanent hypoparathyroidism during primary surgery decreased from $3.2 \%$ in the first period to $0.8 \%$ in the second period $(p<0.001)$. Permanent RLN palsy was found in the time period from 1991-2002 in $0.94 \%(20 / 2,128$ nerves at risk) and from 2003-2010 in $0.88 \%(13 / 1,485$ nerves at risk; $p=0.723)$. Permanent hypoparathyroidism in the time period from 1991-2002 was found in $0.6 \%$ $(9 / 1,482)$ and from $2003-2010$ in $1.1 \%$ (10/928 patients; $p=0.239$ ).

The incidence of recurrent goiter surgery in the first period was $11.1 \%$ and decreased to $8.1 \%$ in the second period $(p<0.001)$. Linear regression analysis showed a significant decrease over the whole study period $(p<0.001$; Fig. 1).

A total of 515 redo operations for recurrent diseases were performed between 1972 and 2010. Seventy percent of recurrent diseases were ipsilateral. This resulted in 360 preventable ipsilateral redo operations, 90 preventable recurrent laryngeal nerve palsies, and 58 preventable malignant diseases with the extended type of resection. Furthermore, 37 preventable radioiodine ablation therapies (radioiodine ablation therapy was necessary in $64 \%$ of these cases [18]) and 15 additionally preventable deaths during our study period of 39 years were associated with the extended technique in the present study population. A model of cost analysis including only objective factors is represented in Table 3.

\section{Discussion}

A significant reduction in permanent recurrent laryngeal nerve palsy and hypoparathyroidism in primary operations was found with extended thyroid resection. The incidence of recurrent goiter surgery was also significantly reduced with this approach. Public health costs are tremendously affected with recurrent diseases.

The prevailing thought until at least 1980s was that hemithyroidectomy or total thyroidectomy was a risky operation. The present results emphasize our previously reported findings [10], now over a period of 39 years, namely that an extended thyroid resection is associated with a significantly reduced rate of permanent nerve palsy and hypoparathyroidism compared to
Table 1 Demographic parameters, indication for primary and redo surgery, and surgical technique

Values in parentheses are percentages

${ }^{\mathrm{b}}$ Values are median (range)

${ }^{a}$ Fisher's exact test unless indicated otherwise

'Student's $t$ test

\begin{tabular}{llll}
\hline & $\begin{array}{l}\text { First study period } \\
1972-1990 \\
n=2,867\end{array}$ & $\begin{array}{l}\text { Second study period } \\
1991-2010 \\
n=2,410\end{array}$ & $p$ value $^{\mathrm{a}}$ \\
\hline Age (years) & $52(15-75)$ & $53(15-83)$ & $0.901^{\mathrm{c}}$ \\
Sex (male/female) & $(20.3 / 79.7)$ & $(19.7 / 80.3)$ & 0.907 \\
Primary surgery & & & 0.587 \\
Benign nodular goiter & $1,631(64.0)$ & $1,400(63.2)$ & 0.123 \\
Hyperthyroidism & $522(20.5)$ & $460(20.8)$ & 0.661 \\
Malignancy & $395(15.5)$ & $354(16.0)$ & 0.828 \\
Redo surgery & & $151(77.0)$ & 0.188 \\
Benign nodular goiter & $249(78.1)$ & $16(8.2)$ & 0.540 \\
Hyperthyroidism & $16(5.0)$ & $29(14.8)$ & \\
Malignancy & $54(16.9)$ & & \\
Typ of resection & 1,673 & - & \\
Subtotal lobe resection & 621 & 925 & \\
Bilateral subtotal resection & 573 & 1,485 & \\
Hemithyroidectomy & - & & \\
Total thyroidectomy & & &
\end{tabular}


Table 2 Outcome parameters

\begin{tabular}{llll}
\hline & $\begin{array}{l}\text { First study period } \\
1972-1990 \\
n=2,867\end{array}$ & $\begin{array}{l}\text { Second study period } \\
1991-2010 \\
n=2,410\end{array}$ & $p$ value $^{\mathrm{a}}$ \\
\hline Hypoparathyroidism & $93(3.2)$ & $19(0.8)$ & $<0.001$ \\
Primary surgery & 2,548 & 2,214 & \\
Nerves at risk & 2,925 & 3,613 & $<0.001$ \\
Recurrent nerve palsy & $105(3.6)$ & $33(0.9)$ & \\
Redo surgery & 319 & 196 & 0.304 \\
Nerves at risk & 563 & 282 & $10(3.5)$ \\
Recurrent nerve palsy & $30(5.3)$ & & \\
\hline
\end{tabular}

subtotal resection. The risk of morbidity was significantly lower in the second study period due to (a) change in general surgical strategy, meaning hemithyroidectomy in all eligible patients with unilateral benign thyroid diseases or total thyroidectomy in bilateral benign disease, and (b) change in surgical technique with obligatory visualization of the recurrent laryngeal nerves and the parathyroid glands. Two meta-analyses showed no difference in the occurrence of permanent complications between subtotal and total thyroidectomy [7, 9]. The significantly lower morbidity rate with the more radical surgical approach in the present study reflects the experience in endocrine surgery at our institution, with over 5,000 thyroid operations performed over a 39-year period.

Subtotal thyroidectomy is associated with recurrent diseases in 7-50 \% [11, 14, 19-21]. However, long-term follow-up is necessary to evaluate correct recurrence rate [9]. In the present study, change in surgical technique led to a significant reduction in the recurrence rate and is a consequence of the more radical thyroid resection in the second study period. There were no differences in permanent recurrent laryngeal nerve palsy in recurrent disease between the two periods. However, reoperation is associated with an increased morbidity rate due to postoperative adhesions compared to primary surgery. This indicates that surgical strategy in the primary operation represents a cornerstone in

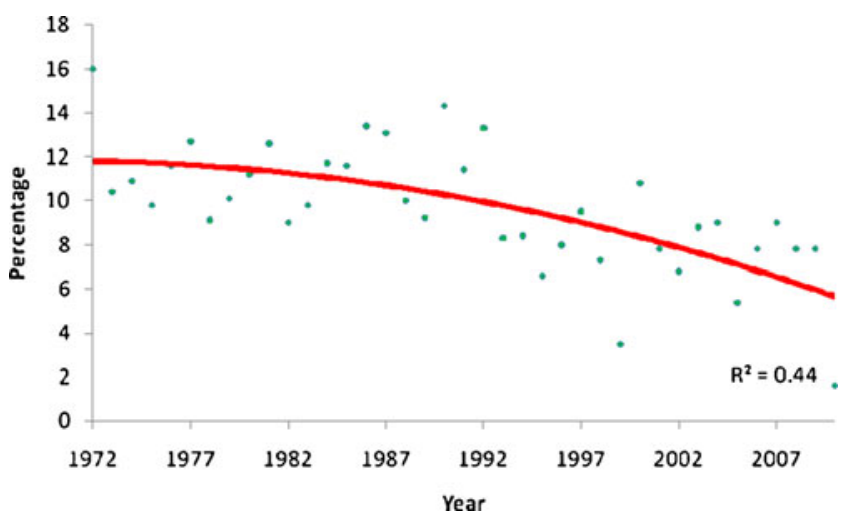

Fig. 1 Redo surgery for recurrent disease. Linear regression analysis shows a significant decrease over the whole study period $(p<0.001)$ preventing redo surgery with its associated high morbidity rate. Furthermore, the threat of bilateral recurrent nerve palsy demanding tracheostomy is probably preventable with the more radical surgical approach.

Recurrent disease is not only associated with a high morbidity rate during redo surgery but also with socioeconomic drawbacks and increased costs. In the present cost analysis model, only objective factors were taken into account. Disadvantages of subtotal resection such as recurrent diseases and high mortality rate were associated with overall costs of 3,790,497.95 EUR. These expenses are equalizing with 99,749.95 EUR savings per year or with hospital costs of 12 thyroidectomies per year over the whole study period of 39 years, respectively. The findings of Crevenna et al. indicated that patients with nonmetastatic well-differentiated thyroid cancer showed significant deficits in health-related quality of life [22]. Furthermore, the adverse effects of 58 preventable cancers in quality of life were challenging to express in direct costs but should be considered in evaluating the above-mentioned costs.

Limitations of the study include lack of randomization. However, long follow-up period and documentation of complication rate in a large series of patients are of potential interest. Intraoperative nerve monitoring was started in 2003. Variation of technical equipment and technique of intraoperative nerve monitoring cannot be excluded over a long time period from 2003 to 2010 . Only patients referred to our clinic were included in the analysis of recurrent disease. However, as a tertiary center specializing in endocrine surgery, the probability that patients with recurrent

Table 3 Model of preventable costs

\begin{tabular}{lrrr}
\hline & Number & \multicolumn{1}{l}{ Costs } & \multicolumn{2}{l}{ Total costs } \\
\hline Thyroidectomy & 360 & $8,190.20$ EUR & $2,948,472.00$ EUR \\
Loss of salary & 360 & $1,504.31$ EUR & $541,551.60$ EUR \\
Recurrent nerve palsies & 90 & $1,278.78$ EUR & $115,090.29$ EUR \\
Radioiodine ablation & 37 & $5,010.38$ EUR & $185,384.06$ EUR \\
Total preventable costs & & & $3,790,497.95$ EUR \\
\hline
\end{tabular}


diseases were treated elsewhere is very low. Cost analysis was performed retrospectively on the basis of the current pay scale, and only objective, well-reproducible factors were included, whereas cost inflations were not taken into account.

\section{Conclusion}

In conclusion, improvements in surgical technique and change in surgical strategy significantly decreased the prevalence of recurrent laryngeal nerve palsy, hypoparathyroidism, and recurrent disease as well as reduced public health costs associated with recurrent goiter. Further studies prospectively investigating costs of socioeconomic factors in thyroid surgery are necessary and will probably be higher than the present calculations.

\section{Conflicts of interest None.}

\section{References}

1. Rutkow IM (1978) William Halsted and Theodor Kocher: "an exquisite friendship". Ann Surg 188:630-637

2. Kennie R (1999) The Nobel surgeon: Theodor Kocher (18411917). J Invest Surg 12:177-178

3. Vitti P, Delange F, Pinchera A, Zimmermann M, Dunn JT (2003) Europe is iodine deficient. Lancet 361:1226

4. Studer H, Gerber H, Zbaeren J, Peter HJ (1992) Histomorphological and immunohistochemical evidence that human nodular goiters grow by episodic replication of multiple clusters of thyroid follicular cells. J Clin Endocrinol Metab 75:1151-1158

5. Bidey SP, Hill DJ, Eggo MC (1999) Growth factors and goitrogenesis. J Endocrinol 160:321-332

6. Paschke R (2011) Molecular pathogenesis of nodular goiter. Langenbecks Arch Surg 396:1127-1136

7. Moalem J, Suh I, Duh QY (2008) Treatment and prevention of recurrence of multinodular goiter: an evidence-based review of the literature. World J Surg 32:1301-1312

8. Dogan L, Karaman N, Yilmaz KB, Ozaslan C, Atalay C (2011) Total thyroidectomy for the surgical treatment of multinodular goiter. Surg Today 41:323-327

9. Agarwal G, Aggarwal V (2008) Is total thyroidectomy the surgical procedure of choice for benign multinodular goiter? An evidencebased review. World J Surg 32:1313-1324
10. Seiler CA, Vorburger SA, Burgi U, Candinas D, Schmid SW (2007) Extended resection for thyroid disease has less operative morbidity than limited resection. World J Surg 31:10051013

11. Barczynski M, Konturek A, Hubalewska-Dydejczyk A, Golkowski F, Cichon S, Nowak W (2010) Five-year follow-up of a randomized clinical trial of total thyroidectomy versus Dunhill operation versus bilateral subtotal thyroidectomy for multinodular nontoxic goiter. World J Surg 34:1203-1213

12. Reeve TS, Delbridge L, Cohen A, Crummer P (1987) Total thyroidectomy. The preferred option for multinodular goiter. Ann Surg 206:782-786

13. Muller PE, Kabus S, Robens E, Spelsberg F (2001) Indications, risks, and acceptance of total thyroidectomy for multinodular benign goiter. Surg Today 31:958-962

14. Barczynski M, Konturek A, Stopa M, Cichon S, Richter P, Nowak W (2011) Total thyroidectomy for benign thyroid disease: is it really worthwhile? Ann Surg 254:724-729, discussion 729-730

15. D'Hubert E, Proske JM (2010) How to optimize the economic viability of thyroid surgery in a French public hospital? J Visc Surg 147:e259-e263

16. Cirocchi R, D'Ajello F, Trastulli S, Santoro A, Di Rocco G, Vendettuoli D, Rondelli F, Giannotti D, Sanguinetti A, Minelli L, Redler A, Basoli A, Avenia N (2010) Meta-analysis of thyroidectomy with ultrasonic dissector versus conventional clamp and tie. World J Surg Oncol 8:112

17. Dralle H, Sekulla C, Haerting J, Timmermann W, Neumann HJ, Kruse E, Grond S, Muhlig HP, Richter C, Voss J, Thomusch O, Lippert H, Gastinger I, Brauckhoff M, Gimm O (2004) Risk factors of paralysis and functional outcome after recurrent laryngeal nerve monitoring in thyroid surgery. Surgery 136:1310 1322

18. Vorburger SA, Ubersax L, Schmid SW, Balli M, Candinas D, Seiler CA (2009) Long-term follow-up after complete resection of well-differentiated cancer confined to the thyroid gland. Ann Surg Oncol 16:2862-2874

19. Scerrino G, Salamone G, Farulla MA, Romano G, Salamone S, Pompei G, Buscemi G (2001) Non-toxic multinodular goitre: which surgery? Ann Ital Chir 72:647-651, discussion 652

20. Rios A, Rodriguez JM, Galindo PJ, Montoya MJ, Canteras M, Parrilla P (2005) Surgical treatment of multinodular goiter in young patients. Endocrine 27:245-252

21. Bellantone R, Lombardi CP, Boscherini M, Raffaelli M, Tondolo V, Alesina PF, Corsello SM, Fintini D, Bossola M (2004) Predictive factors for recurrence after thyroid lobectomy for unilateral non-toxic goiter in an endemic area: results of a multivariate analysis. Surgery 136:1247-1251

22. Crevenna R, Zettinig G, Keilani M, Posch M, Schmidinger M, Pirich C, Nuhr M, Wolzt M, Quittan M, Fialka-Moser V, Dudczak R (2003) Quality of life in patients with non-metastatic differentiated thyroid cancer under thyroxine supplementation therapy. Support Care Cancer 11:597-603 